\title{
Blockchain Technology as a Risk Mitigation Tool in Supply Chain
}

\author{
Mian Wasim Layaq, Alexander Goudz, Bernd Noche, Muhammad Atif \\ Department of TUL, University of Duisburg-Essen, Duisburg, Germany \\ Email address: \\ mian.layaq@uni-due.de (M. W. Layaq), alexander.goudz@uni-duisburg-essen.de (A. Goudz), \\ bernd.noche $a$ uni-duisburg-essen.de (B. Noche), atif.muhammad $a$ stud.uni-due.de (M. Atif)
}

\section{To cite this article:}

Mian Wasim Layaq, Alexander Goudz, Bernd Noche, Muhammad Atif. Blockchain Technology as a Risk Mitigation Tool in Supply Chain. International Journal of Transportation Engineering and Technology. Vol. 5, No. 3, 2019, pp. 50-59. doi: 10.11648/j.ijtet.20190503.12

Received: September 24, 2019; Accepted: October 4, 2019; Published: October 15, 2019

\begin{abstract}
The main purpose of this paper is to investigate the research development in supply chain risk management (SCRM) and to connect the new emerging digital technology Blockchain that could help to mitigate risks in any supply chain. Emerging literature, case studies, citations are used to fulfil this the task. The study will explore Blockchain and supply chain risks in details and will compare, how Blockchain based supply chain can mitigate these risks. There are many risks associated to supply chains but we will limit our study to only supply risk and study different areas of Blockchain application in supply chain with supply risk. Blockchain technologies have a potential to upgrade current supply chains. This will not only increase its performance in terms of speedy flow of raw material, finished goods, information and money flow but also help to mitigate current risk in supply chain by increasing visibility and transparency of supply chain. Smart contracts, IoT and Blockchain can serve together to achieve maximum efficiency and effectiveness in SCM. This research contributes to explore further research fields of Blockchain technology in different areas of supply chain risk management.
\end{abstract}

Keywords: Blockchain Technology, SCM, Supply Chain, Supply Chain Risk Management, Supply Risk, IoT, Smart Contracts

\section{Introduction}

The concept of this research is to understand the emerging digital technology of Blockchain and its possibilities in the field of logistics and supply chain management and in which aspects it can help in risk management. The implications of technology probably change supply chain operations and risk involved with them. With the emerging of digital technology record keeping and accessing of stored information have changed the modern world in every aspect of life. Sharing of information is one of main advantages of internet. But till now original papers with sign and stamps are needed to process our legal and official work in all over supply chain because information shared with internet is not immutable and is controlled by some central authority. Blockchain is a new emerging technology and a lot of research is being carried out for its extensive application.

Risk management is the focus of this research. Risk management is used to plan and avoid possible risk scenarios with traditional strategies. Risk management is methodically distinguishing risk to a supply chain and creating approaches to limit them. It decides the most vital dangers to your process and to choose how you dispense assets to manage them. Traditionally risk management is all about identifying, analysing and evaluating possible risks. After these different strategies are used to minimize and mitigate risks. In risk management strategies, one the strategy is 'Planning Reaction to Minimize Effects' which include the approach for risk outcome that is to mitigate the effect of risk. In the scope of this research, we will see that how Blockchain will mitigate the risks in supply chain.

Three main research questions to investigate in this research

1. What is Blockchain from different perspectives?

2. What are the "Supply Risks" involved in supply chain?

3. How Blockchain can have an impact to mitigate risks in supply chain? 


\section{Methods}

\subsection{Blockchain}

Blockchain is a tamperproof and irreversible database, which is distributed along the network, record information in a continuously growing basic unit called block [1]

Blockchain is based on the idea of distributing data in a network rather than saving it on a central storage point. Blockchain can be described as distributed digital ledger, which record digital information and share it to a distributed network. [2] Nakamoto presented idea of Bitcoin based on Blockchain which is, a distributed ledger technology.

As Blockchain is in its development phase, there is no uniform standard definition every author as defined in different ways. There are some selected definitions.

Blockchain is a peer-to-peer network that work on top of internet to transfer ownership hence value without using central control. [3].

Blockchain is a special form of Distributed ledger technology with some extra feature to serve as database. This database is distributed and shared which is also encrypted. Blockchain is tamperproof, irreversible, and hacking proof [4].

The study [5] define Blockchain conceptually as a peer-topeer network which stores database on every node, which is transparent and distributed. A lot of researchers and users are taking interest in it globally. Many from them are sure that it has a potential to comprehensive change in many domains. [6]

After some consensus mechanism transaction is approved and encrypted with hash function and recorded with time of processing in form of block. Each block is then connected with respective hash. Once the block is attached to the chain, it is no longer possible to reverse it [1].

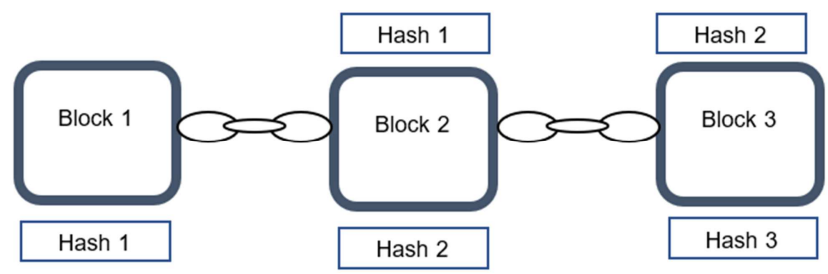

Figure 1. Block connected by Hash header and footer ${ }^{l}$.

A Blockchain record information in blocks and use cryptography to chain these blocks in chronological order or sequence i.e. each block can be traced back using hash number [7]. During the creation of a block, each or some selected nodes can use some consensus method to approve a block to add it in the Blockchain [5].

In order to understand the Blockchain application scenarios and getting its real potential we must use indigenous capabilities of the Blockchain. These capabilities are, a: shared, b: secure ledger and c: distributed [8].

According to [9] Blockchain can be divided in to three main categories depending on basic method of use, which are explained below.

\subsubsection{Public Blockchain}

It is referred to a Blockchain, which is free to access and write transactions in it, and have authority to verify other transactions and can participate in consensus. Anyone with an internet and computing device can enter in this Blockchain. This type of Blockchain are permission less as these are open to everyone. Bitcoin Blockchain and Ethereum are some examples. [9]

\subsubsection{Private Blockchain}

In contrast to public a Blockchain, the private Blockchain is not open for everyone. Participant nodes are limited to only authorized people. As every new entering node in a private Blockchain needs a permission of other members who are already in Blockchain. This feature allows data to limited to members and it fulfil the privacy aspect, which make it use for private organizations. Therefore, this is also referred to as enterprise Blockchain. [10]

\subsubsection{Consortium Blockchain}

This type of Blockchain can be public or private Blockchain but some nodes are preselected only these nodes are able to validate the transactions. This group of people can only participate in consensus. So, this is categorized in permissioned Blockchain. This Blockchain can also control the access of history of Blockchain it may restrict to a specific group or all participants [11]. This is contradictory with the basic idea of Blockchain that protocol is open and for everyone. But as validation or consensus process of early Blockchain is slow and expansive this type of Blockchain provide the solution which is partly decentralized. Some industries are developing consortium Blockchain like Hyperledger. We will explain Hyperledger later [9].

\subsubsection{Smart Contract}

Idea of smart contract was first presented by Nick Szabo in 1994 and is defined as "computer programmed protocol that execute the agreed terms of a digital contract". The idea was to write executable codes, which contain clauses of contract and these codes are stored on a property that can autonomously implement these clauses. Resulting in minimizing or removing the need of third party's mediation to enforce the terms of contract [12].

Smart contracts came more into practice with the development of Blockchain technology, these smart contracts are translated into programme and stored on the Blockchain. Storage of this contract have a unique address by doing a transection into this address will execute this smart contract. Smart contracts allow Blockchain to use functions and conditions beyond monetary exchange for example validation of assets. Ethereum is the first ever Blockchain which allows to use smart contracts [13]. Some authors refer this version of Blockchain as a Blockchain 2.0. These new versions of contracts have the potential to disrupt every type of business and many more [14].

Smart contracts inherit some basic benefits over normal 
working environment related to the contacts for binding and its enforcement. Most prominent are accuracy, efficient operations, cost of operations and transparency of system etc. which allows its applications in multiple areas. Smart contracts are also vulnerable to security and operational risks because these are irreversible than communication failure between nodes. Hacking or viruses may lead to certain consequences [14].

Smart contract provides possibility to do contract in very less time and gives flexibility for a very short period as well, which is not possible in the traditional contracts. After the successful execution of a contract, remittance can be transferred automatically which is cost efficient. Smart contract also gives freedom to sign a contract without personal presence or posting original document from one party to another. This gives also freedom to do a contract beyond the traditional international boundaries as it is only done virtually. Decentralized operation of smart contract on Blockchain does not need intermediator, as it executes autonomously, it eliminates the need of a lawyer [15].

\subsubsection{Internet of Things (IoT) and Blockchain}

Internet of things (IoT) can communicate with others and can exchange data, commands and information but this information is not stored at all or not in sequence. Blockchain can not only record but secure data cryptographically by making it protected from reversing and changing. For example, an IoT connected device is in warehouse, smart container or in a smart home can have information regarding temperature or location [16], this information can be transferred to a Blockchain. This information on a Blockchain will be secured and shared decentralized about the state and status of a smart packet, a smart pallet or a smart container during transportation and keeping its records to match with the terms and conditions of the contract. [17] [18]. Smart contracts can also be integrated to execute terms of contract, for example due to a change in the temperature of a product, which could not be any more useable. By executing the term "write off items" is worthy at that time rather than transporting and spending money on a faulty product. [19]

Conventional devices, when connected with IoT are capable of work in a smart environment and take decision autonomously. New digitization technologies are giving this concept a reality. Blockchain is the key digitization technology which has transformed the information sharing concept by making it more secure and decentralized. IoT industry has already got major disruption by digitization technologies like big data and cloud computing. Blockchain can further enhance its limits. The internet of things (IoT) was first used as a Wireless Sensors Network (WSN) Technology, which is now evolved to Radio Frequency Identification (RFID) that gives it the capacity to communicate data via internet [20]. Which gives it the freedom to potentially use any electronic device from a hardware to wearables. The IoT allows to interact and exchange data with each other like virtually connected world, which represents the real world [14].

IoT can be installed on containers, which will enable container to communicate with other devices and this container can be referred as a smart container. Smart container can be traced with GPS in real-time, allowing more customer satisfaction and at the same time, it will be able to connect with entities of a container terminal and sharing information to custom authorities.

Following information can be integrated digitally in a smart container and the smart container can integrate digital information i.e. location of container, contents of container, condition of container (temperature, damage etc), destination of container, transporting schedule and transporting history etc.

The IoT combination with supply chain management can be achieved by adding a digital tag, which is an equipment with a built-in smart chip. This electronic tag can communicate its ownership, warranties, contents, location or any other special data. Along with this, Blockchain can document that data with integrity and efficiency [21].

In the year 2019, IBM wins "NexGen Supply Chain Leadership Award for Blockchain and IoT" [22]. Supply chain transformation is an initiative of IBM to focus on transformation of future supply chain with the use of Blockchain. The IoT technology use case was basic use case among two others "Parts Provenance" and "Custom Declaration". IoT Blockchain in supply chain can provide the traceability of products throughout it's life cycle and possibility to trace products in real time. "Parts provenance" gives the complete outlook of parts and steps at which they are integrated in product as well as its origin. This provide authentic data for compliance and risk management. "Customs Declaration" during import and export is an important but time-consuming blockage of international goods. Compliance risks can be reduced by increasing visibility. Also, by digitization of the processes will reduce the risk of possible delays in receiving original papers to the process. This will give visibility of product in various outlook like product life cycle management and supply chain risk management [23].

\subsection{Supply Chain Risk Managment}

With the development of new technologies, in every field of businesses, there is regular need to update supply chain to match the pace of industries and provide solutions to new problems or make competitive edge. Businesses are going global in order to gain economic benefits by producing goods in developing countries which made supply chain more complex. Due to fluctuation in businesses' requirements, supply chains are in great pressure. Supply chain is required to be proactive to detect and act quickly against any disruption or even possibility of disruption.

Supply chain is a system which contains elements like suppliers, manufacturers, distributers, retailers and customers and these elements are connected for material, finance and information flow in both directions [24]. Or in other view, supply chain is a series of operations and activities, which are 
needed to move products from raw material to finished goods to the end users [25].

Supply chain is much more than just transportation of goods. Outsourcing is most common trend in supply chains in which products as services are purchased from other companies local as well as international. Which may further outsource some or all of its parts. Which make complete supply chain venerable to many risks. Procurement is now an important field in supply chain to manage outsourcing.

For international supply chain, there are many operations involved. And these operations are carried out by various organizations. Right after manufacturing, manufacturer itself or an intermediator, export the manufactured goods to international customer, which may use it as a part of other product (i.e. assembly part of automobile) or sale directly to whole sale dealers and retailers. During transportation, a lot of paper work is involved like export invoices, air way bills, packing lists, bill of lading etc. And transportation is not only in one step, mostly, intermodal transport is carried out to transfer goods across the globe, which further involves, shipping companies to provide services on land and sea. To shift the mode of transportation, loading and unloading services are also needed. These services are further subcontracted. Before loading and after unloading, authorities are needed to custom in order to evaluate duty on the products. Elsewise, the insurance companies are also important parties, as most of the products are insured during the transportation, which provide insurance claims for mishaps during transportation. All these processes need optimum control of information. These services need to be paid according to their services and product cost is also paid by importer to exporter. Other cost depends upon how the parties have agreed to pay, there are many incoterms used in industry. In figure 2, flow of information and payments are displayed as a traditional practice in supply chain.

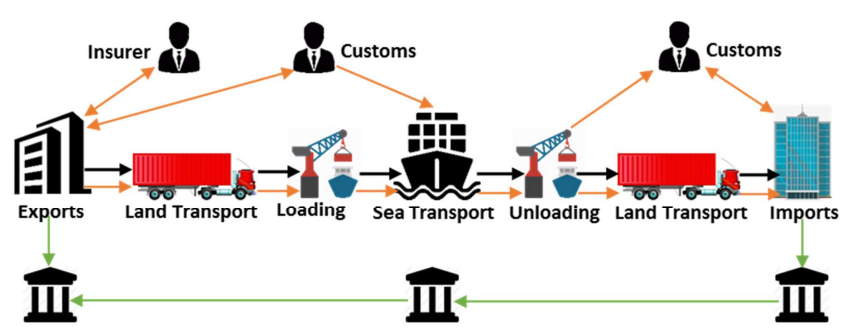

$\longrightarrow$ Flow of goods $\longrightarrow$ Flow of Information $\longrightarrow$ Flow of Payments

Figure 2. Traditional practice of supply chain ${ }^{2}$.

\subsubsection{Risk}

Risk cannot be defined in specific words; it is the probability of the unforeseen occurrence of something, which can have a negative impact on any Business. In general, the supply chain risk is considered to have negative impact as it is important to manage negative risk in order to gain maximum productivity rather than studying positive risks, which are already in favour of industry.

\subsubsection{Risk Definitions from Different Perspectives}

Different authors have defined risks differently; some important definitions are given below:

Risk is a direct measure of probability of its occurrence and its potential degree of damage that can take place by selecting the given risky situation [28].

Risk is the possible chances of taking place of an uncertain outcome or situation that have negative effects on results of a project [29].

A "quantified chance of occurrence of a specific hazard is known as risk" [30]

Risk can be defined in many ways, but the most general definition can be as, risk is outcome of likeliness of an event to occur at a certain time and location which has the possibility to adversely impact on the organization [31].

Risk is the level of exposure to adversely affect the situation that is needed to understand and managed in order to control the business objectives [32]. Risk is part of every business especially when going globally for outsourcing supply chains, it becomes more risky and vulnerable to many types of risks. More and more businesses are taking risk management more seriously [33].

Supply chain of today is more complex and challenging as it was never before, with businesses going globally, there are different mode of transportations involved especially for international supply chains. As companies are manufacturing in economical part of world and sell in other parts of the world to gain maximum commercial advantages. This leads to relatively long supply chains, which are quite challenging and costs a lot of time and resources. Slight change in demand and disturbance in scheduling can lead to a high cost change. At the same time, this can also lead to different type of risks like penalties, loss of perishable items, delays for goods in lean manufacturing, where goods are planned for just in time etc. Other type of risks, which are more common nowadays is traceability of goods for transportation and for its entire lifecycle. This traceability of product can give confidence to user about its originality, genuineness and origin. Moreover, in the future of digitalization of "Industry 4.0 ", supply chain will be more complex and there is a need to develop new ways to integrate with "Industry 4.0".

Anything in supply chain that disturb the flow of information or material from base supplier to end customer is considered as risk in supply chain [34].

\subsubsection{Types of Risks}

Risks in supply chain can be categorized into two main types, a. External risks b. Internal risks.

Examples of external risks are supply risks, procurement risks, geopolitical risks, business risks, environmental risks, demand risks, economic risks etc.

Internal risk examples are manufacturing risks, Material flow risks, process risks, technological risk, cultural risks, control risks etc [35]. 


\section{Results}

\subsection{Blockchain-Based Supply Chain and Its Effect on Supply Risks}

With the introduction of the new industrial revolution, "Industry 4.0", supply chain needs to be upgraded to match the core requirement of "Industry 4.0". This new version of supply chain may be called as "Supply chain 4.0 ". There are many digitization technologies, which can be integrated to develop the new generation of supply chain.

Supply chain is growing in complexity from many other digitization technologies. Blockchain has the ability to integrate all elements of supply chain and record data in decentralized manner. This will provide transparency between members with the ability to trace back by recording all the information-flow e.g. material, products, and finances. This information is verifiable and gives ability to trace the origin and complete lifecycle of the product.

Supply chain needs to be sustainable. Blockchain implementation in supply chain can help to achieve it. Blockchain can give the advantage of reducing number of intermediator or eliminate them, hence its cost will be reduced. The improved version of document sharing will increase the speed by reducing the waiting time and time needed to sort out information from original documents and labels. Traceability is a plus point of this technology. Combination of new digitization technologies in supply chain can give the competitive superiority among competitors. Innovation in supply chain is needed to realize the benefit of new technologies [26].

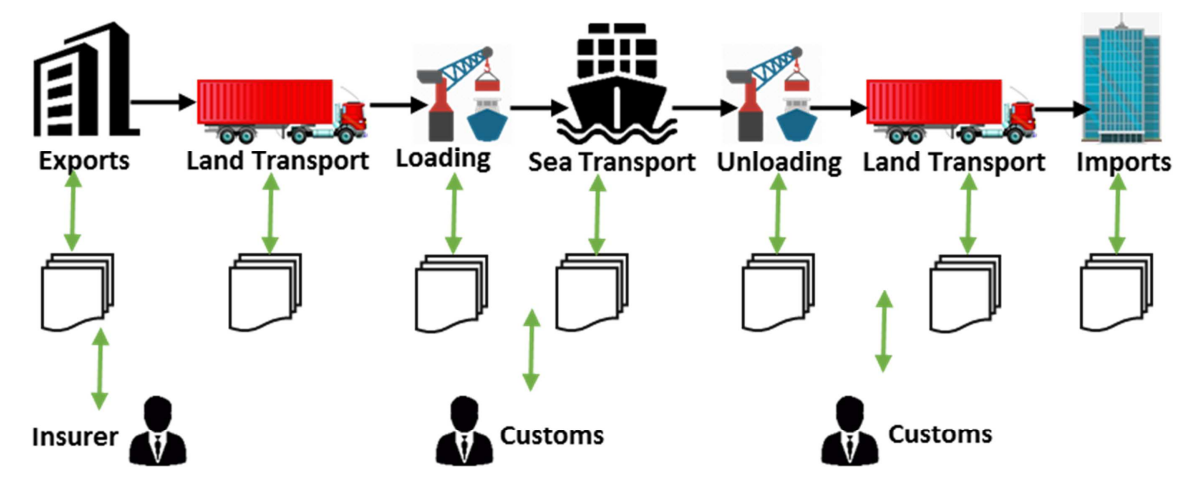

$\longrightarrow$ Flow of goods $\longrightarrow$ Every one is updating and retrieving required information and payments

Figure 3. Possible Supply chain using Blockchain concept.

Future supply chains need to be faster, more flexible, more gradual, more accurate and more efficient. Faster in a sense to reduce overall delivery time and this is only possible by advanced forecasting approach for both external and internal data as well as status of supplies. Amazon is already one step ahead. It holds a patent "predictive shipping" in which products are shipped to the required location even if the order has not been placed. After placement of order, shipment can be directed to the delivery address. To achieve such a high flexibility, rerouting should be possible even in the middle of the way. More customer specific production will be possible with the need of new transport concepts. Accurate and Realtime information of supply chain will be in demand which should be more efficient [27].

Use cases are being developed in different areas of supply chain by integrating main features of Blockchain. There are solutions of many current problems in supply chain with Blockchain. Most common applications' focus is on problems like tracing of product during complete supply chain from the beginning to the end and during transportation. The flow of products can be more visible by using Blockchain technology with IoT, this visibility of product during flow, provides more control, flexibility and optimized flow of product, during complete supply chain. This Visibility can also show quality parameters of product at every step, which provides better quality assurance then controlling the quality at the end of production and having some sort of surprise. Demand forecasting can be more accurate when real data is available in real time which is more authentic as well. Smart contracts in Blockchain can execute transactions automatically without inter mediators, which will reduce risk of fraudulent transaction, so as dispute between parties. Implementation areas of Blockchain in supply chain, when used in combinations, can be tracing back the origin of product and its flow in the supply chain.

The study [36] have categorised Blockchain properties into two main characteristics, which are having an important impact on logistics, supply chains and their networks.

Decentralized information exchange in Blockchain is cryptographically secured, easy to access at downtime and also trusted, verifiable and provide real time information to every authorised members of supply chain [36].

Automation of transaction is possible with smart contracts which execute an agreed set of instructions and can also automatically verify the transactions [12].

Decentralized information exchange is a fixed base of many supply chain use cases, almost every area of supply chain can use this feature to improve overall service of supply chain. Moreover, smart contract gives extra feature to automate transactions, which together with decentralized processes add extra combined benefits.

The strengths of Blockchain have an ability to affect every 
type of supply chain risk. As it provides a strong base for autonomous working in Blockchain, which can provide a better collaboration without trusting any one. Blockchain can provide that needed trust to make everyone accountable for their every action as it will maintain record of each transaction of communication as well as monetary.

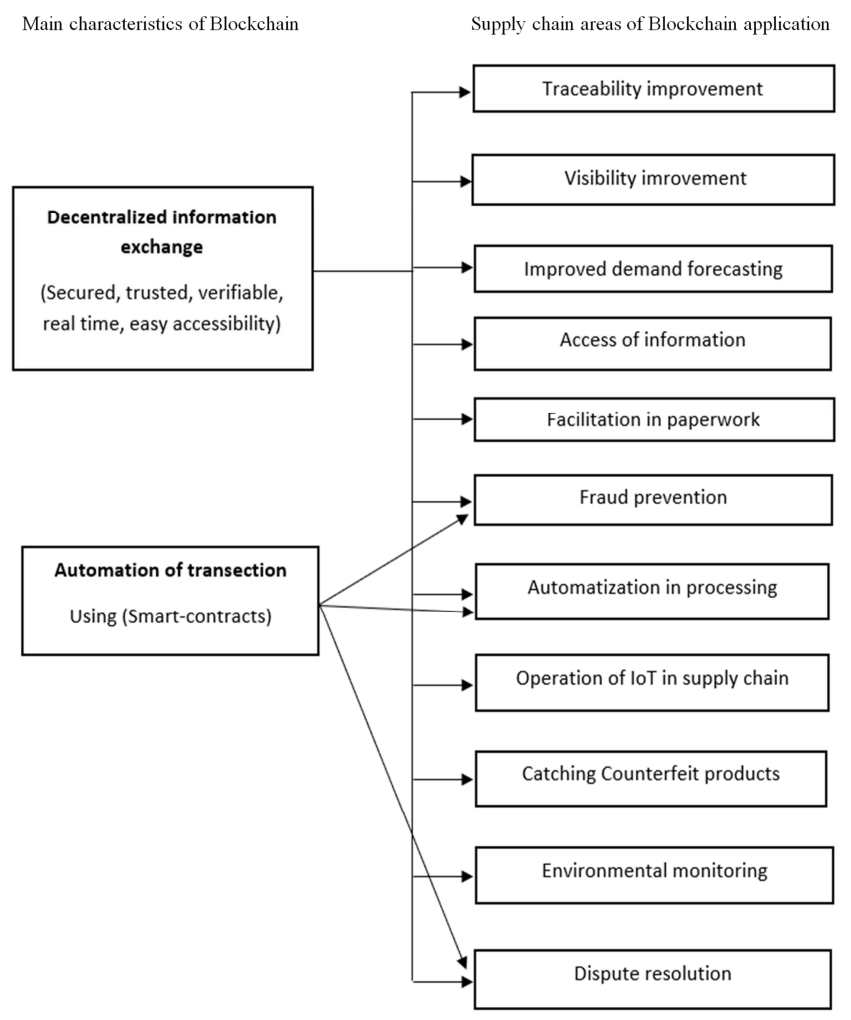

Figure 4. Blockchain implementation areas in supply chain ${ }^{3}$.

\subsection{Traceability Improvement}

Traceability of a product is very important, and it is directly related to supply risk, if the origin of the product is traced and tracked accurately, it will provide information of exact manufacturing data of the product like place of production, date of production and suppliers involved. Tracking of origin of products is also important in food supply chain in which it is required to keep records like which product is coming from which field and which fertilizers are used for it. Due to this level of tracking, if there are affected items then they are identified and removed from the market. This calling back is applied only for affected product rather than the complete batch of products.

Blockchain due to its tamperproof record provide an excellent reference for tracing back the origin of material or components used in the manufacturing of products. It also provides information that which supplier have produced it and at which time. In Blockchain based supply chain, digital labels are used rather than traditional barcodes, which stores and communicate information digitally.

For example, parts and components of an assembly are sourced worldwide from three different suppliers and assembled at a location and delivered to a customer. After delivery to the customer, problem is detected in a part of the assembly. Due to strong traceability, it is possible to identify the supplier of a part in relatively short time. Time is very important, as it will lead to the next supply as well with the same fault.

\subsection{Visibility Improvement}

Visibility of supply chain is a key to have a strong control on managing the flow of supply chain. Supply chain is more visible, when each movement of supply network is visible in real time. Due to the high visibility of supply chain, more cost and service optimization is possible.

In supply chain with Blockchain technology, IoTs can be installed on container, pallets, or even on packaging. These IoTs turn normal container to a smart container, which can communicate digitally with other devices and store all these communications on Blockchain.

Due to this communication, smart containers can trace during the entire supply chain phase and making the supply chain highly visible. Not only this, it also stores all the movement in a trusted record. This Realtime tractability reduce the supply risk of late arrival.

High visibility can be extended to production process, where, each process can be recorded on Blockchain, which can make quality assurance more effective. This can inform the quality aspects of product before the product is shipped from the supplier. In case of faulty product which may be rejected after delivery to customer, can be identified and risk related to supplier failure can be mitigated.

In supply risk, the procurement risk is one of the important risks, which can be considerably mitigated with high visibility in supply chain. Supplier compliance data can be stored on Blockchain, which, with the help of a smart contract can be automatically audited. Whenever the certifications of the suppliers are expired or there is a change in process on the supplier end, that needs to be validated. Smart contract can be executed and automatically identify the non-conformed products. This can also help in automated supplier evaluation.

\subsection{Improved Demand Forecasting}

Demand management is an important part of supply chain, which also affect the supply risk and when the forecasting is accurate, it will mitigate supply risk of short production or over production. [36]

Forecasting always depend on the historical data which is used to forecast for next period. Accuracy of the forecast is based on the most recent and trusted historical data. Blockchain provides the most recent data in real time and trusted secured history up to the first block. Also due to access of information on Blockchain, data related to the product is available for every participant, which leads to batter planning. 


\subsection{Access of Information}

In Blockchain, depending, which type of Blockchain is used to access the information can be different. In supply chain, there is a need of collaboration of many organizations and at the same time some sort of data are also needed be secured. Collaboration on Blockchain relation to data exchange is very secure and efficient.

Efficient and timely information exchange can reduce many supply risks like inventory information at supplier's end or in the storage of warehouse, can lead to timely planning for an alternative resource.

\subsection{Facilitation in Paperwork}

During containerized shipping, there are a lot of documents involved, which needs excessive paper work from start till the end of the transportation, which costs time and money. Other than that, these documents are needed to be shipped in original ahead of shipment, so that these original documents can be presented on Port. These documents of shipping trail are also at risk prone i.e. loss of documents, fake documents or changed documents.

\subsection{Fraud Prevention}

In Blockchain, by giving verification of source of origin or authenticity of the product, prevent fraud of fake products. Production of product can be stored on Blockchain with their unique identifications, which then later provide reference to verify the authentication.

This function of Blockchain can reduce supply risk by adding quality-controlled parts on Blockchain, which is then verified automatically at assembly station. This will ensure the quality of the assembled product.

\section{Discussion}

Beside the above results, the Blockchain technology could have a significant impact on the improvement of the whole SCM process, below are some more processes, which could be vitally improved if merged with Blockchain.

\subsection{Automation in Processing}

Smart contract gives the power to Blockchain to automize many processes. Once the conditions are met in the code of the smart contract, it will execute the agreed conditions automatically. The major benefit of smart contract is that there is no need of central authority to ensure contract terms like banks or courts.

[37] discussed the idea of using smart contract enabled Blockchain in supply chain and logistics. In the first step, supply chain contract and conditions related to it are translated into an executable code and this code is stored on Blockchain. This contract code can access Blockchain network. Once the contracted parties fulfil all the required obligations, contract will be executed and transactions will be settled, for example, leasing of a smart crane to transfer smart container ${ }^{4}$ from truck to warehouse. Once the container is transferred, contracts will be executed, and funds will be transferred.

Automated transactions are naturally faster and more cost effective and at the same time more error proof as compare to human's data entry or communication to other humans. Due to autonomous working environment, better utilization of resources is possible. It will also remove or minimize the intermediators, which along with economic benefits also improves the supplier customer relationship.

Due to fast communication in supply chain, visibility will increase the probability of on-time shipments that will ultimately reduce supply risk. Automatic transfer of funds, once the job is considered as completed, which in this case will be fast and will reduce the risk of shortage of financial resources.

\subsection{Operation of IoT in Supply Chain}

As supply chains are also using sensors and IoT to identify the product and its status in network in real time. It will generate a lot of data, which need to be stored accordingly with time. Blockchain can solve this problem by recording status and sharing it to the required members [38].

Operation of IoT in supply chain can be better in function with Blockchain technology. As it is the foundation of "Supply chain 4.0" and without this, the idea of "Industry 4.0 " is not feasible and it also have an impact on the supply risk.

In traditional supply chain, batch production is unavoidable, and batches of certain quantity are required to operate economically. But with "Industry 4.0", batch size can be reduced to one piece. [39]

Because of making small batch size, economical supply risks can be mitigated. This will make supply chain more agile and adaptation of new product design will be very fast. Products can meet the trend and reduce the out of trend supply in market.

\subsection{Catching Counterfeit Products}

In compliance, it is necessary to identify and remove the counterfeit product. These products can adversely affect the consumer or may be these are parts of another product. Counterfeit products can have a large destructive impact on reputation of the company.

In medical supply chain, counterfeit medicine is becoming problematic. Pharmacies are in a liability to ensure originality of the drugs to be sold and make their network secure for entering any fraudulent or expired drugs.

Reputation is a major supply risk in current social media age. For example, a company is using a small component from a supplier and this supplier's reputation is maligned due to some reason. This bad reputation of supplier can also affect the business of its customer. Blockchain can ensure to catch counterfeit products at basic step.

4 Smart crane and smart container are IoT enabled containers 


\subsection{Environmental Monitoring}

Digital supply chain will be using many smart devices i.e. smart containers, smart carriers etc. These devices can also record environmental conditions inside and outside of the container or carrier. This environmental data can be very useful for taking many environmental related decisions and regulatory complaints i.e. $\mathrm{CO}_{2}$ emissions.

Monitoring of environment within container will record temperature and humidity and store it on a Blockchain with time and variation in reading. This stored data can then be used later as a verification to the required environmental conditions, agreed with any logistic company.

This control of environment gives strength to supply chain to deliver the products at desired temperature, in order to ensure the quality of product and in some cases, this change in temperature may result in loss of goods, which is surely a supply risk. These types of risks are typically related to food items or animal transportation. Surely, when the product is wasted during transportation, this will lead to shortage of goods at required place, which can adversely affect the business.

With Blockchain, this change in temperature can be timely detected and actions can be taken to reduce the risk. Actions like improving temperature condition before it increases certain critical value, where product is wasted. And, if the product is already wasted, there is no need to transport waste to its destination and it can be discarded at the same point rather than burning more resources on transporting wasted material. On other side an alternate route can be time triggered in order to mitigate the risk due to short of supply.

\subsection{Dispute Resolution}

Blockchain records each transaction more securely which can provide a strong reliable reference for sorting out dispute in supply chain. As many parties are involved in supply chain and furthermore, a lot of communication is needed, which some time may generate dispute.

Dispute may be due to violation of terms or bad service in terms of lost goods or damaged goods. Which may lead to claim management, which in traditional supply chain is also complex. Insurances must investigate the main reason of loss. For example, a container of sea food is shipped and on arrival to the customer this sea food is rotten. It will be hard to investigate at which point during transportation temperature was raised.

Blockchain can record temperature during complete journey of time and this data is shared to every party involved and Blockchain can also integrated with smart contract, which will be automatically executed and act as a violation of contract and penalties are enforced as agreed before.

\section{Conclusion}

Blockchain is a decentralized ledger of record of ownership of an asset that can record and monitor any type of transaction. This record is distributed decentralized across the network. Transactions are stored in form of cryptographically locked blocks and these blocks are arranged in a sequence of their generation. Alteration in the block is very hard, which make it secure.

Although Blockchain was first developed to introduce digital currency but Blockchain technology possess key features, which can be used to solve many present problems in both financial and non-financial sectors. This technology is not completely matured yet as it is still in the development phase. Blockchain technology can improve efficiency in many sectors and enhance globalisation.

Many different use cases are developed using Blockchain technology and there is also continuous research in adoption of Blockchain technology. Some supply chain companies have different Blockchain use cases even in the testing phase of supply chain areas. Focus is on visibility, transparency and paper trail of shipping industry.

Supply chains today are more vulnerable than in the past due to growing globalisation. Due to cheap labour in overseas, many companies are manufacturing or sourcing goods internationally. This leads to many types of risks in supply chain. These risks need to be identified and need to be properly managed with risk management process. In risk management process, risks are treated with proper techniques to mitigate the risk. Blockchain technology can be a tool to mitigate risk. Blockchain may not completely over through the current supply chain technologies. But integrating its basic features, like method of data storage and retrieval, automation of processes into the current supply chain processes can get some benefits to mitigate risk and improve performance. Other than this, a Blockchain layer can also be used on top of current supply chain to get competitive advantage by reducing vulnerabilities.

Blockchain can have effect on every type of risk in supply chain and in most of the cases, it tends to mitigate risk or ultimate removal of the risk. Adoption of Blockchain may also generate new type of risks, which are currently not present during the adoption phase. Other new risks can be improved, as technology gets more mature.

Flow of supply chain can be dramatically disrupting, when supply of the system is disturbed and it is due to supply risks. These risks can be reduced by having a real-time information of flow entities of supply chain and it is only possible by increasing the visibility of the entities.

With the implementation of Blockchain in supply chain and logistic, many areas of performances can be improved i.e. visibility, traceability, demand forecasting, access of information etc. Improved performances in these areas can reduce vulnerabilities of supply risks.

\section{Future Work}

Blockchain applications in supply chain can have impact on all types of risks in supply chain. Future work is to explore, how Blockchain can influence on other types of risks. 
1. As Blockchain is in the initial phase in supply chain, aspect of risk managements could be integrated in development or improvement of Blockchain applications.

2. Blockchain applications in controlling manufacturing processes and advance manufacturing.

3. Blockchain applications in collaboration and integration of global manufacturing locations.

4. Blockchain technology applications in production assembly of large machines industry i.e. Trains, Planes, Automobile.

\section{References}

[1] M. Swan, Blockchain: Blueprint for a new economy, O'Reilly Media, Inc., 2015.

[2] Nakamoto, Satoshi, "Bitcoin open source implementation of P2P currency," 2008. [Online]. Available: http://p2pfoundation.ning.com/forum/topics/bitcoin-opensource. [Accessed April 2019].

[3] Marco Iansiti and Karim R. Lakhani, "The Truth About Blockchain," 2017. [Online]. Available: https://hbr.org/2017/01/the-truth-about-blockchain.

[4] Wright, Aaron and De Filippi, Primavera, "Decentralized Blockchain Technology and the Rise of Lex Cryptographia," 2017. [Online]. Available: https://papers.ssrn.com/sol3/papers.cfm?abstract_id=2580664. [Accessed April 2019].

[5] F. Glaser, "Pervasive Decentralisation of Digital Infrastructures: A Framework for Blockchain enabled System and Use Case Analysis," 2017.

[6] R. C. M.-B. a. J. L. K. Beck, "Governance in the Blockchain Economy. A Framework and Research Agenda," 2018.

[7] A. V. S. N. U. a. G. F. Schweizer, "Unchaining Social Businesses - Blockchain as the Basic Technology of a Crowdlending Platform," 2017.

[8] Pablo Junco, "Accelerating the adoption of enterprise blockchain," 9 Nov. 2017. [Online]. Available: https://azure.microsoft.com/en-us/blog/accelerating-theadoption-of-enterprise-blockchain/. [Accessed April 2019].

[9] I.-C. L. a. T.-C. Liao, "A Survey of Blockchain Security Issues and Challenges," International Journal of Network Security, Vol. 19, No. 5,, pp. 653-659, 2017.

[10] Dr. Shermin Voshmgir, "Blockchain, Smart Contracts und das Dezentrale Web,” TECHNOLOGIE STIFTUNG BERLIN, 2016.

[11] Vitalik Buterin, 6 Aug 2015. [Online]. Available: https://blog.ethereum.org/2015/08/07/on-public-and-privateblockchains/. [Accessed April 2019].

[12] Konstantinos Christidis, Michael Devetsikiotis, "Blockchains and Smart Contracts for the internet of things," IEEE, pp. 2292-2303, 2016.

[13] V. Buterin, "A Next-Generation Smart contract and Decentralized Application Platform," April 20192013. [Online]. Available: https://github.com/ethereum/wiki/wiki/White-Paper.
[14] C. M. J. C. E. S. M. D. Ana Reyna, "On blockchain and its integration with IoT. Challenges and opportunities," ELSEVIER, 2017.

[15] Hergueta, Roberto Fernandez, "Making Blockchain Real in Transport \& Logistics," 8 May 2017. [Online]. Available: https://www.slideshare.net/roberhergueta/20170425-makingblockchainreallogistics. [Accessed April 2019].

[16] A. K. S. J. R. Dorri and P. Gauravaram, "Blockchain for IoT Security and Privacy: The Case Study of a Smart Home," IEEE International Conference on Pervasive Computing and Communications Workshops, pp. 618-623, 2017.

[17] M. S. O. P. N. M. P. S. M. a. A. Y. Z. D. Puthal, "Secure and Sustainable Load Balancing of Edge Datacenters in Fog," IEEE Communications Magazine, 2017.

[18] D. P. S. S. S. P. M. a. A. Y. Z. S. Ray, "Building a Sustainable Internet of Things," EEE Consumer Electronics Magazine, pp. 42-49, 2018.

[19] D. \&. M. N. \&. M. S. \&. K. E. \&. D. G. Puthal, "Everything You Wanted to Know About the Blockchain: Its Promise, Components, Processes, and Problems," IEEE Consumer Electronics Magazine. 7, pp. 6-14, 2018.

[20] Manuel Díaz, Cristian Martín, Bartolomé Rubio, "State-ofthe-art, challenges, and open issues in the integration of Internet of things and cloud computing," Journal of Network and Computer Applications Vol. 67, pp. 99-117, 2016.

[21] Don Tapscott, Alex Tapscott, Blockchain Revolotion, How the technology behind bitcoin is changing money, busim'ness and the world, New York: An imprint of Penguin Random House LLC, 2016.

[22] NextGen Supply Chain, "Announcing the 2019 Nextgen Supply Chain Award Winners," 1004 2019. [Online]. Available: https://nextgensupplychainconference.com/2019/04/announcing -the-2019-nextgen-supply-chain-award-winners/. [Accessed May 2019].

[23] IBM, "IBM's Global Supply Chain Transformation Wins 2019 NextGen Supply Chain Leadership Award for Blockchain and IoT," $2019 . \quad$ [Online]. Available: https://newsroom.ibm.com/2019-04-17-IBMs-Global-SupplyChain-Transformation-Wins-2019-NextGen-Supply-ChainLeadership-Award-for-Blockchain-and-IoT.

[24] Fiala, P., "Information sharing in supply chains," The International Journal of, pp. 419-423, 2005.

[25] C. Donald, J. Waters, Supply Chain Risk Management: Vulnerability and Resilience in Logistics, London. UK: Kogan Page Limited, 2007.

[26] Mikita Lushcheuski, "Problems in achieving consensus in sustainable supply chain formation," South-Eastern Finland university of applied sciences, 2019.

[27] Mckinsey \& Company, "Supply Chain 4.0 - the nextgeneration digital supply chain," Oct. 2016. [Online]. Available: https://www.mckinsey.com/businessfunctions/operations/our-insights/supply-chain-40--the-nextgeneration-digital-supply-chain. [Accessed April 2019].

[28] Lowrance, W. W., "The Nature of Risk'in Schwing, RC and Albers, WA (eds.) How Safe is Safe Enough," Plenum Press, New York. NY, 1980. 
[29] P. H. D. a. N. K. Simon, " Project Risk Analysis and Management Guide," Association for Project Management, Norwich, 1997.

[30] Brindley Clare, Supply Chain Risk, Hampshire: Ashgate Publishing Limited, 2004.

[31] H. Kerzner, Project Management: a systems approach to planning scheduling and controlling 9th ed, New Jersey: John Wiley \& Sons, 2006.

[32] Deloach J. W., Enterprise-Wide Risk Management: Strategies for Linking Risk \& Opportunity (Financial Times Management Briefings), London: Financial Times/Prentice Hal, 2000.

[33] Jim Lawton, “http://blog.sourcinginnovation.com/," 2007. [Online]. Available: http://blog.sourcinginnovation.com/2007/02/14/five-types-ofsupply-risk-and-how-to-mitigate-them.aspx. [Accessed April 2019].

[34] Norrman, A. and Lindroth, B., "Supply Chain Risk Management: Purchasers' vs. Planners' Views on Sharing Capacity Investment Risks in the Telecom Industry.," in 11th International IPSERA conference, Enschede, The Netherlands, 2002.

[35] Crefield, "Understanding Supply Chain Risk: A SelfAssessment Workbook Centre for Logistics and Supply Chain Management at the Cranfield School of Management," 2003. [Online].

[36] D. Dujak, D. Sajter, "Blockchain Applications in Supply Chain," in SMART Supply Network, Cham, Springer, 2019, pp. 21-43.
[37] DHL \& Accenture, "BLOCKCHAIN IN LOGISTICS: Perspectives on the Upcoming imact of blockchain technology and use cases for the logistics industry," DHL Customer Solutions \& Innovation, Troisdorf, Germany, 2018.

[38] Niels Hackius and Moritz Petersen, "Blockchain in logistics and supply chain: Trick or treat?," in Digitalization in Supply Chain Management and Logistics, Hamburg, epubli, 2017, pp. $3-18$.

[39] Claudia Witte, "Batch size 1 will soon cost no more than mass production," 14 Dec 2017. [Online]. Available: https://www.hannovermesse.de/en/news/batch-size-1-willsoon-cost-no-more-than-mass-production-62402.xhtml. [Accessed April 2019].

[40] Antony Lewis, "A Gentle Introduction To Blockchain Technology," 29 Oct 2015. [Online]. Available: https://bravenewcoin.com/insights/a-gentle-introduction-toblockchain-technology. [Accessed April 2019].

[41] Microsoft, "how-blockchain-will-transform-modern-supplychain," $2018 . \quad$ [Online]. Available: https://azurecomcdn.azureedge.net/cvtfa07c1602f20d76da45dee719c203732fba17981e 7a2c5f4e0a 7 $790 \mathrm{cfb} 0 \mathrm{a} 4 \mathrm{a} 6 \mathrm{c} / \mathrm{mediahandler/files/resourcefiles/how-}$ blockchain-will-transform-modern-supply-chain/howblockchain-will-transform-modern-supply-chain.pdf. [Accessed April 2019].

[42] Luke Parker, "Bank of America Merrill Lynch explores using a blockchain for trade finance," 01 Mar 2016. [Online]. Available: https://bravenewcoin.com/insights/bank-ofamerica-merrill-lynch-explores-using-a-blockchain-for-tradefinance. [Accessed April 2019]. 